\title{
La terminologie grammaticale russe et ses sources grecques
}

Sylvie Archaimbault

\section{OpenEdition}

1 Journals

Édition électronique

URL : https://journals.openedition.org/hel/649

DOI : $10.4000 /$ hel. 649

ISSN : 1638-1580

Éditeur

Société d'histoire et d'épistémologie des sciences du langage

Édition imprimée

Date de publication : 30 juin 2021

Pagination : 73-90

ISBN : 9791091587143

ISSN : 0750-8069

\section{Référence électronique}

Sylvie Archaimbault, "La terminologie grammaticale russe et ses sources grecques », Histoire Épistémologie Langage [En ligne], 43-1 | 2021, mis en ligne le 28 octobre 2021, consulté le 29 octobre 2021. URL : http://journals.openedition.org/hel/649; DOI : https://doi.org/10.4000/hel.649

\section{(c)}

HEL is licensed under a Creative Commons Attribution-NonCommercial-NoDerivatives 4.0 International License 


\title{
LA TERMINOLOGIE GRAMMATICALE RUSSE ET SES SOURCES GRECQUES
}

\author{
Sylvie Archaimbault \\ CNRS, Cultures et sociétés d'Europe orientale, balkanique et médiane \\ (UMR8224, Eur'ORBEM), Paris, France
}

\begin{abstract}
Résumé - La terminologie grammaticale russe résulte d'une synthèse des terminologies grecque et latine qui s'est opérée au début $\mathrm{du} \mathrm{XVII}^{\mathrm{e}}$ siècle dans la grammaire slavonne de Meletius Smotrickij. Cependant, pour des raisons historiques, culturelles et religieuses, le socle grec a été fondateur. Cet article retrace la genèse de la terminologie dans les principaux traités grammaticaux du slavon. Centré sur la dénomination des parties du discours, il accorde une attention particulière au statut de l'article, catégorie superflue au regard des faits de langue, qui apparaît comme un marqueur de la fidélité au modèle grec.
\end{abstract}

Mots-clés — alphabet, baptême de la Russie, grammaire, parties du discours, russe, Russie ancienne, slavon
Abstract - The Russian grammatical terminology stems from a synthesis of Greek and Latin terminologies, which occurred in the early 17 th century in the Slavonic grammar by Meletius Smotrickij (1618). However, for historical, cultural and religious reasons, a Greek base was dominant. This paper aims at retracing the genesis of the terminology in the main grammatical treatises of the Slavonic language. It pays particular attention to the status of the article while focusing on the naming of the parts of speech. We claim that the article, a superfluous category with regard to the facts of the Slavonic language, appears as a marker of fidelity to the Greek model.

Keywords - alphabet, ancient Russia, baptism of Russia, grammar, parts of speech, Russian language, Slavonic language

\section{Au COMMENCEMENT ÉTAIT L'ALPHABET}

Il suffit d'aborder les origines de la pensée grammaticale dans les terres slaves pour qu'une évocation s'impose, celle des deux frères, Cyrille (Constantin) et Méthode, natifs de Salonique et créateurs d'alphabets. À cette évocation vient s'adjoindre le sentiment d'un lien entre l'outillage de la langue, sous la forme de l'écriture tout d'abord, de la grammaire ensuite, et l'adoption d'une religion. Cette double référence a pris la valeur d'un mythe fondateur dans la conscience russe. Les plus anciennes chroniques russes, réunies dans la célèbre Chronique de Nestor ${ }^{1}$, ou Récit des temps passés (Povest'vremennyx let), que l'on peut qualifier

1 Nestor est un moine lettré, ayant vécu au début du XII siècle, auteur d'une réélaboration de chroniques historiques antérieures fragmentaires. Ce récit épique consiste en une « réécriture de l'histoire russe [qui] 
de fondatrice, confondent les différents peuples en un seul et même peuple slave et glorifient la venue des frères en terre slave, conséquence directe de l'évangélisation des Slaves :

Il n'y avait qu' une seule race slave ${ }^{2}$ : et ces Slaves qui étaient établis sur le Danube et qui furent soumis par les Ougres étaient les Moraves, les Tchèques, les Polonais et les Polianes, que l'on appelle maintenant Rus'. C'est pour eux, les Moraves, qu'a été pour la première fois inventé l'alphabet que l'on appelle alphabet slave ; ce même alphabet se trouve aussi chez les Rus' et les Bulgares du Danube. (Arrignon $2008: 85$ )

Ce sont les princes slaves, rapporte la chronique, qui ont dépêché leurs ambassadeurs auprès de l'empereur Michel et sollicité de Byzance l'envoi de religieux lettrés pour traduire les textes sacrés :

Notre pays est baptisé et nous n'avons pas de maître pour nous prêcher, nous instruire et nous expliquer les livres saints. Nous ne comprenons ni la langue grecque, ni la langue latine; les uns nous instruisent d'une façon, les autres d'une autre. Aussi nous ne comprenons pas le sens des livres sacrés, ni leurs forces. Envoyez-nous donc des maitres qui soient capables de nous expliquer la lettre des livres sacrés et leur esprit. Ayant entendu cela, l'empereur Michel rassembla tous ses théologiens et leur transmit tout ce qu'avaient dit les princes slaves. Et les théologiens dirent : À Thessalonique, il y a un homme, nommé Léon. Il a des fils qui connaissent la langue slave et deux d'entre eux sont de fins théologiens. (ibid. : 86)

Ces deux fils sont Méthode et Constantin le philosophe, qui prendra le nom de Cyrille en devenant moine, peu avant sa mort :

Dès que les frères arrivèrent, ils commencèrent à composer un alphabet slave et traduisirent les Actes des Apôtres et l'Évangile. Et les Slaves furent heureux d'entendre la grandeur de Dieu en leur langue. Ensuite ils traduisirent le Psautier, l'Octoèque et d'autres livres. (ibid. : 87)

Dans cet événement fondateur que fut l'évangélisation des Slaves, Rome aurait été prise de court par Byzance. Le baptême de la Russie, en 988, représente un aboutissement dans des relations déjà bien établies avec Byzance, même si elles n'étaient pas toujours sereines. Il existe dans les sources byzantines de nombreuses mentions des Slaves orientaux et notamment, de leur incursion guerrière

s'inscrit donc clairement dans une vision cohérente de construction de l'État kiévien à l'époque de Jaroslav le Sage (1019-1054). Les Russes sont en mesure d'assumer tout à la fois leur baptême, qui a fait d'eux un peuple élu et victorieux, et leur pouvoir, qui s'organise au sein d'une fratrie dynastique semblable à celle que constituent les fils de Noé. En ce sens, le Récit des temps passés témoigne de sa très grande envergure : il est une véritable construction historico-religieuse destinée à donner une identité commune aux tribus slaves désormais unifiées dans un puissant État centralisé, dont Kiev est le centre religieux, politique et culturel. Le Récit des temps passés, instauré en référent identitaire, se veut un texte fondateur». La traduction de la chronique que nous citons est celle de Jean-Pierre Arrignon (2008).

2 La question de l'unité slave est restée tout à la fois vivace et âprement débattue. D'un point de vue linguistique, elle renvoie à l'étude du slave commun, langue mère hypothétique, qui aurait conservé son unité jusqu'à une date tardive. Meillet indique que des preuves linguistiques du haut degré d'unité des dialectes slaves sont notables jusqu'au IX ${ }^{\mathrm{e}}$ siècle. Même après cette date, et nonobstant des modifications, " les unes n'ont pas changé la structure générale des mots, et les autres ont eu lieu parallèlement dans les diverses langues, si bien que le résultat final n'a pas trop différencié les langues slaves les unes des autres » (Meillet 1910 : 533). 
dans Constantinople. Comme le note Viktor Živov (2017 : 81), on possède des informations avérées sur la présence chrétienne dans la Russie kiévienne au $\mathrm{X}^{\mathrm{e}}$ siècle $^{3}$, et ce avant même le baptême de la Russie.

La Vie de Méthode, texte rédigé peu après la mort de Méthode, en 885, par l'un de ses disciples, fait état de la rivalité entre les églises. Il est fait mention dans ce texte d'une lettre que le pape Adrien II aurait adressée au prince morave Rostislav - Byzance a devancé Rome, au terme d'une rivalité d'influence :

Ce n'est pas seulement, en effet, auprès de ce siège hiératique [le Saint-Siège] que vous avez demandé un éducateur, mais aussi auprès du pieux empereur Michel et il vous a envoyé le bienheureux Constantin le Philosophe avec son frère, avant que nous n'y soyons parvenus. (Knjazevskaja 1999 [XI ${ }^{\mathrm{e}}$ siècle] : VIII, 38 ; Vaillant $1968: 38)$

Ainsi le lien avec la culture grecque byzantine est-il affirmé comme un fondement de l'identité culturelle et historique slave en général, russe en particulier ${ }^{4}$. Cette césure religieuse a structuré le monde slave en une Slavia romana, catholique et attachée à l'alphabet latin, et une Slavia orthodoxa, avec son alphabet parent de l'alphabet grec et sa liturgie donnée directement en grec, avant de l'être en slavon (Picchio 1963). Au demeurant, de nombreuses traces directes de la langue grecque se retrouvent dans des inscriptions ou dans des textes. Le grec était la langue de l'église en Crimée, tout au moins entre les $\mathrm{IX}^{\mathrm{e}}$ et $\mathrm{XI}^{\mathrm{e}}$ siècles. Enfin, Byzance a longtemps fourni métropolites et évêques (Comtet $2002: 225$ ).

Ce sentiment d'un lien organique avec la culture grecque a alimenté aussi une certaine mythologie des origines de la grammaire. Il se trouve ainsi un recueil grammatical datant de 1620, consistant en une copie de la grammaire slavonne de Laurent Zizanius, qui se place directement sous l'autorité des créateurs d'alphabet et grammairiens gress, fussent-il mythiques : Livre nommé Grammaire de la langue slave, composé par de sages anciens, Palamède et Kadmos ${ }^{5}$ et Denys le grammairien (Kuz'minova 2002).

Les premiers témoignages de la pensée grammaticale des slaves sont attribués par les chercheurs à Jean l'Exarque, prélat bulgare de la fin du $\mathrm{IX}^{\mathrm{e}}-$ début du $\mathrm{X}^{\mathrm{e}}$ siècle, proche du tsar bulgare Siméon (893-927), brillant disciple de Méthode et traducteur de nombreux textes du grec en slave. Les introductions à ses propres traductions lui fournissent l'occasion d'exposer des choix qui mettent en cause

3 Pour une synthèse récente de ces questions encore très discutées aujourd'hui, on se reportera à l'ouvrage posthume de Viktor Živov (2017 : 76-97).

4 Ces différents témoignages écrits passent sous silence l'existence de deux alphabets: l'alphabet cyrillique, dont la parenté avec l'alphabet grec est patente, et l'alphabet glagolitique, ou glagolite, d'une apparence différente, qui rappelle l'alphabet copte, mais structurellement proche du cyrillique. La primauté des alphabets continue à faire l'objet de débats, on trouvera une bonne synthèse récente de ceux-ci chez Olivier Azam (2001). Jean Breuillard et Stéphane Viellard mettent en exergue le caractère " performant » de ces deux alphabets, car « remarquablement adaptés à la phonologie de la langue pour laquelle ils ont été créés » (Breuillard \& Viellard 2015 : 29). Je tiens à remercier Stéphane Viellard d'avoir bien voulu m'autoriser à reproduire la table de présentation des deux alphabets (ibid. : 28).

5 Palamède est l'inventeur mythique des échecs, de l'ordre des batailles ; il est dit par Théophraste avoir inventé les chiffres et les lettres. Kadmos, fils d'Agénor, roi de Tyr, fondateur de la ville de Thèbes fut considéré comme l'adaptateur en Grèce de l'alphabet phénicien. 
Tableau 1 - Tableau comparatif des alphabets repris à Breuillard \& Viellard (2015 : 28)

\begin{tabular}{|c|c|c|c|c|c|}
\hline glagolite & cyrillique & dénomination vieux-slave & glagolite & cyrillique & dénomination vieux-slave \\
\hline 中 & $\boldsymbol{a}$ & $\mathbf{a} 3 \mathbf{b}$ 'je, moi' & • & $\phi$ & $\phi \rho \mathbf{k T h}$ \\
\hline 世 & $\mathbf{E}$ & воук'ы 'lettre' & b & $X$ & $x^{\mathrm{tk} \rho \mathrm{z}}$ \\
\hline$\vartheta$ & B & $\begin{array}{l}\text { в'At' et вłAи } \\
\text { 'connais' }\end{array}$ & $\boldsymbol{V}$ & ц & ци \\
\hline 9 & $\boldsymbol{\Gamma}$ & Глаголи 'dis' & 4 & YY & чрьвь 'le ver' \\
\hline$\delta$ & $A$ & Аовро 'le bien' & Ш & Ш & ша \\
\hline $\mathbf{3}$ & $\boldsymbol{E}$ & 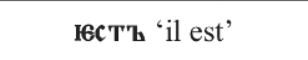 & ய' & భ & $\Psi a$ \\
\hline み & ж & $\begin{array}{l}\text { живњте } \\
\text { ‘vous vivez' }\end{array}$ & न्व & $\mathbf{b}$ & $\begin{array}{l}\text { 16pł Voyelle ultra-brève } \\
\text { d'arrière (proche de [ə]) }\end{array}$ \\
\hline 8 & s 7 & stkno 'très' & $\begin{array}{l}98 \\
\text { gिक }\end{array}$ & $\begin{array}{l}\mathbf{b l} \\
\mathbf{b u}\end{array}$ & геры \\
\hline$\theta^{\circ}$ & 3 & земмга 'la terre' & .9 & $\mathbf{b}$ & $\begin{array}{l}\text { 16ph voyelle ultra-brève d'avant } \\
\text { (proche de [i]) }\end{array}$ \\
\hline 8 & $\mathbf{I}$ & $\begin{array}{l}\text { I 'i décimal', } \\
\text { représentant } 10\end{array}$ & $\Delta$ & 古 & IaTb \\
\hline M & (h) & г'єрвь & & Ia & /a/ jodisé, /ja/ \\
\hline$\alpha$ & Н и & $\begin{array}{c}\text { ижє } \\
\text { ‘qui’, représentant } 8\end{array}$ & $\mathbb{P}^{P}$ & $\boldsymbol{1 0}$ & /u/ jodisé, /ju/ \\
\hline 3 & K & какө 'comme' & & 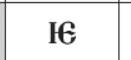 & /e/ jodisé /je/ \\
\hline 8 & $\Lambda$ & пюдию 'le peuple' & $€$ & $\mathbf{A} \mathbf{A}$ & petit jus /ę/ \\
\hline भా & $M$ & машь 'notre' & 2E & $\mathbf{H A}$ & petit jus jodisé /ję/ \\
\hline$P$ & $\mathbf{N}$ & мъыслите 'pensez' & अ€ & x & grand jus /Q / \\
\hline 9 & $\bullet$ & ONł 'celui-là' & $\Delta E$ & 1 & grand jus jodisé /jǫ/ \\
\hline 10 & П & пюкөи ‘le repos’ & & בุa & ӑ્и [ksi, lettre grecque] \\
\hline $\mathbf{b}$ & $\rho$ & рьци 'dis' & & 卌 & 廿и [psi, lettre grecque] \\
\hline $\mathbf{8}$ & c & $\begin{array}{c}\text { сльвь } \\
\text { 'la parole, le verbe' }\end{array}$ & $\theta$ & $\theta$ & ита $[$ fita $=$ thêta grec $]$ \\
\hline - & $\mathbf{T}$ & $\begin{array}{l}\text { тврьвА॰ } \\
\text { 'fermement' }\end{array}$ & 8. & $v$ & ижица [ižica = upsilon grec $]$ \\
\hline $\boldsymbol{g}$ & or 8 & oүk' & (\$) & $\mathbf{w}$ & WT'k 'de' \\
\hline
\end{tabular}


des différences linguistiques, ou au contraire, des similitudes, entre les langues. Ces observations linguistiques l'amènent à privilégier en traduction « la force et l'esprit » de la langue, plutôt que la littéralité, ce qui fait dire à Kolesov que Jean l'Exarque avait développé une véritable "théorie ouverte de la traduction» (Kolesov 1991 : 208). Si Jean a énormément traduit, on lui a aussi beaucoup attribué. Ainsi de la traduction d'un traité grammatical inachevé de Jean Damascène, désormais appelé « pseudo-Damascène », car ces deux attributions sont l'une et l'autre fautives. Le traité pseudo-Damascène est en fait une compilation, traduite beaucoup plus tardivement.

Le $\mathrm{XI}^{\mathrm{e}}$ siècle a été marqué par une période intense de traductions des textes grecs en slavon ${ }^{6}$. La disparition du premier Empire bulgare, devenu une province byzantine en 1019 suite à la conquête de Basile II, dit Basile le Bulgarochtone (tueur de Bulgares), avait poussé nombre de clercs bulgares vers Kiev, où une importante école de traduction s'ouvrit près de Sainte-Sophie. La Chronique de Nestor témoigne de cette importante activité, encouragée par le prince Jaroslav le Sage :

Jaroslav $^{7}$ aimait les établissements religieux ; il aimait aussi beaucoup les prêtres et tout particulièrement les moines. Il s'intéressait aux livres et les lisait souvent nuit et jour. Il réunit de nombreux copistes et fit des traductions du grec en langue slave; il écrivit et rassembla beaucoup de livres à travers lesquels les vrais croyants ont pu s'instruire et se réjouir de l'enseignement de Dieu. (Arrignon $2008: 265)$

Si les traductions mises en avant sont celles de textes religieux, d'autres avaient aussi pour objet des textes civils. Comme cela se vérifie souvent, une telle pratique répandue de traduction a accompagné l'essor d'une culture philologique et grammaticale. La méthode du calque à partir de termes érudits grecs a été largement utilisée tout d'abord pour la désignation des figures de rhétorique, dans la traduction du traité Des tropes de Georges Choeroboscos au XI ${ }^{\mathrm{e}}$ siècle (Kolesov 1991 : 209-210). Vingt-sept catégories sont identifiées, dont l'allégorie [inoslovie], la métaphore [prevod], la synecdoque [sopriatie]. Ces traductions mettent en œuvre le calque et créent l'habitude de recourir à cette méthode, qui sera largement exploitée par la suite dans les textes grammaticaux.

Dans les traductions des textes, le calque ne se contente pas de toucher le lexique, il peut s'étendre à l'organisation de la phrase, pour réaliser des calques syntaxiques, comme le relève Živov :

Transposer purement et simplement en texte slave la syntaxe d'un original grec ou latin était tout simplement impossible, bien que ces langues fussent parentes et

6 Le terme «slavon » traduit le russe цүерковнославянский язык (littéralement, langue slave d'église). Il s'agit de la langue écrite, d'un registre élevé, caractéristique des textes religieux. Viktor Živov fait à ce terme russe deux reproches: celui de gommer les emplois dans des textes non religieux et celui de méconnaître les différentes variantes locales de cette langue élevée (bulgare, serbe, macédonien, croate, tchèque...). Le terme français offre la possibilité de nommer ces variantes, puisque l'on parle d'un slavon serbe, d'un slavon russe, etc. Pour ce qui est de la Russie, cette langue élevée cohabite avec le vieux-russe, écrit ou parlé. Cf. Živov $2017: 77$.

7 Jaroslav le Sage (978-1054), successeur de Vladimir I ${ }^{\mathrm{er}}$ (Saint), grand-prince de Kiev (958-1015), régna de 1019 à 1054 . 
qu'il y eût dans la syntaxe beaucoup en commun. Toutefois, il était possible de conserver l'ordre des mots, aussi libre en slave que dans les langues classiques, on trouvait des équivalents adéquats pour les conjonctions et particules grecques pour coordonner et subordonner les propositions. Dans les cas où la langue vernaculaire n'offrait pas de correspondance, il restait à copier la construction syntaxique grecque. C'est pourquoi dans les traductions slavonnes, on trouve quantité de calques syntaxiques, comme par exemple le double accusatif, calquant l'Accusativus [duplex] du $\operatorname{grec}^{8}$. (Živov $\left.2017: 24-25\right)$

On voit là la puissance des effets de la traduction dans un moment d'élaboration d'une langue savante, qui se développe alors dans une relation de dépendance par rapport au modèle grec.

\section{L’ÉLABORATION D’UNE TERMINOLOGIE GRAMMATICALE POUR LE SLAVON}

Les ouvrages qui ont jalonné l'édification de ce cadre terminologique sont des traités grammaticaux restés manuscrits jusqu'à leur édition par Vatroslav Jagić au $\mathrm{XIX}^{\mathrm{e}}$ siècle $^{9}$, ainsi que des grammaires slavonnes, publiées pour la plupart au tournant des $\mathrm{XVI}^{\mathrm{e}}$ et $\mathrm{XVII}^{\mathrm{e}}$ siècles, dans des milieux très ouverts à la culture grecque. Ils prennent place dans un contexte historique et culturel sur lequel nous reviendrons brièvement.

Il importe de rappeler que suite à différentes incursions guerrières, la principauté de Kiev s'est trouvée défaite et disloquée, partagée en trois grandes parts : les pays dits du Sud-Ouest, Ukraine et Belarus actuels, qui passent sous domination lituanienne puis polono-lituanienne; les principautés de l'Ouest, Novgorod et Pskov, ouvertes sur la Hanse ; et celles de l'Est, soumises au joug tatar (Moscou, Vladimir, Tver). Les pays du Sud-Ouest prirent alors le relais de la culture kiévienne, en une double opposition au catholicisme polonais d'une part et à l'orthodoxie moscovite de l'autre. Avec la soumission polonaise, l'opposition des deux religions et des deux cultures a trouvé à se revivifier, ainsi que les deux traditions grammaticales : la grecque orientale et la latine catholique. Et comme la réflexion grammaticale ne trouvait pas sa place dans les monastères de Moscovie, où était proscrit l'enseignement des sciences, elle s'épanouit aux marges occidentales, dans les villes de Lvov, Vilna ou Kiev. Outre d'importants centres intellectuels, celles-ci hébergeaient des foyers de résistance à la polonisation et à la catholicisation, regroupés en des sortes de guildes ou confréries de

8 En écrivant ici Accusativus simplex, Viktor Živov commet un lapsus calami, que nous avons rectifié.

9 Vatroslav Jagić (1838-1923), philologue croate, élève de Franz Miklosich, a été le premier titulaire d'une chaire d'études slaves à Berlin entre 1874 et 1880 . En raison de ses sympathies pour le mouvement panslave, il accepta à plusieurs reprises des postes dans les universités de l'Empire de Russie, à celle de Nouvelle-Russie (située à Odessa) de 1872 à 1874, puis à celle de Saint-Pétersbourg, où il remplaça Izmail Sreznevskij comme professeur de slavon et de russe, à partir de 1880. Au sein de l'Académie impériale des sciences de Russie, il engagea un important chantier d'inventaire et de publication des sources grammaticales manuscrites dédiées au slavon, organisant une vaste collecte des documents conservés dans les bibliothèques publiques et privées, ainsi que dans les monastères de la Slavia orthodoxa, soit en Russie et dans les pays slaves du Sud. Ce travail a été publié sous la forme d'un imposant volume intitulé, Réflexions de l'Antiquité slave du Sud et russe sur le slavon (Jagič 1968 [1896]). 
laïcs (bratstva), qui luttaient pour préserver une relative autonomie religieuse et transmettre des savoirs et savoir-faire artisanaux.

Les grammaires accordent peu de place aux faits de langue vernaculaire et décrivent en très grande part la langue des textes religieux, un slavon assez homogène, sans faire droit aux particularités locales et aux disparités, graphiques notamment. Cette homogénéité doit beaucoup aux rédacteurs des grammaires qui marchent dans les pas de leurs prédécesseurs et entretiennent le dessein d'une unité slave, linguistique et religieuse. Pour trouver décrits les faits de la langue usuelle, il faut chercher dans les descriptions élaborées par des étrangers, missionnaires ou commerçants, soucieux de partager la connaissance d'une langue vivante et de promouvoir ainsi un outil de compréhension et d'échange avec les populations. Ces dernières descriptions (Mark Ridley, 1594-1599; Tönnies Fenne, 1607; Heinrich Wilhelm Ludolf, 1696 ; Jean Sohier, $1724^{10}$ ) mettent en avant les différences existantes entre le slavon et le russe. Comme le résume un peu brutalement, en une formule qui est passée à la postérité, le piétiste allemand Wilhelm Ludolf, dans sa grammaire publiée à Oxford en 1696, «C'est pourquoi on dit chez eux [les Russes] que l'on parle en russe et que l'on écrit en slavon ${ }^{11}$. Ceci bien que Ludolf lui-même s'intéressât de façon comparée au russe et au slavon, mais aussi et surtout, bien qu'une langue vieux-russe écrite fût attestée, comme l'ont montré par exemple les découvertes archéologiques menées dans les années 1950 à Novgorod. Les écrits sur écorce de bouleau retrouvés redonnent vie au dialecte novgorodien $\mathrm{du} \mathrm{XII}^{\mathrm{e}}$ siècle, ce qui amène le célèbre linguiste récemment décédé Andrej Zaliznjak (1933-2017) à remettre en cause les représentations admises de l'histoire de la langue russe, basée jusqu'alors exclusivement sur l'étude des textes littéraires et religieux. L'apprentissage du slavon dans les écoles religieuses a encouragé la diglossie dans les couches élevées de la population, situation que Boris Uspenskij décrit comme transitoire. En effet, accompagnant le développement d'une culture écrite russe, le bilinguisme, qui suppose un choix conscient des locuteurs entre deux systèmes linguistiques maîtrisés, supplante la diglossie (cf. Uspenskij 1994 : 10-53 ; 1997 ; 2002).

Les descriptions grammaticales de la langue vernaculaire participent à la diffusion d'une langue russe de communication, en dehors du pays. Ces premières grammaires vernaculaires, dont la connaissance s'est considérablement enrichie à la fin du $\mathrm{XX}^{\mathrm{e}}$ siècle, excèdent notre sujet. Elles n'innovent pas vraiment sur le plan de la terminologie, mais reprennent le schéma des grammaires grecques et latines en usage. Nous nous contenterons ici d'étudier la terminologie des grammaires du slavon.

Celles-ci sont donc tout d'abord des grammaires fragmentaires restées manuscrites, qui ont été publiées par Vatroslav Jagić entre 1885 et 1895 . Et ce sont ces

10 On dispose pour ces différentes grammaires d'éditions reprographiées réalisées au XX ${ }^{\mathrm{e}}$ siècle : Ridley 1996 [1594-1599] ; Fenne 1970 [1607] ; Ludolf 1959 [1696] ; Sohier 1987 [1724].

11 Ludolf 1959 [1696], Prafatio $A^{2}$ : «Adeoque apud illos [Russos] dicitur, loquendum est Russice \& scribendum est Slavonice». Roger Comtet (1999) replace la Grammatica russica de Ludolf dans son contexte historique et culturel, tout en s'intéressant au message porté par le corpus d'exemples et de maximes traduits par l'auteur et regroupés en fin de volume. 
grammaires qui ont forgé le moule terminologique dont on peut mesurer, encore aujourd'hui, l'extraordinaire continuité. Pour des raisons qui tiennent à l'histoire et à la culture religieuse, le fonds grec a constitué un socle aussi ancien que durable, même si la terminologie grammaticale russe est au final le produit d'une certaine hybridation entre les traditions grammaticales grecque et latine.

Le traité le plus ancien publié par Jagić est intitulé Des huit parties de l'énoncé [O osmix častex slova]. Il date du début du XIV e siècle, décrit des faits de langue hétéroclites et apparaît comme une compilation de plusieurs sources byzantines réalisée en Serbie et ayant pénétré en Russie via la Bulgarie et la Moldavie (Worth 1983 : 14). Il bénéficia d'une certaine notoriété puisqu'il servit de modèle à un manuscrit plus tardif, du $\mathrm{XV}^{\mathrm{e}}$ siècle, passé à la postérité sous le nom de Damascène [Damaskin] et qui connut une grande diffusion ${ }^{12}$. Cette attribution erronée lui a valu le nom de pseudo-Damascène [lže-Damaskin], qu'il conserve parfois encore aujourd'hui. Ce traité est à la base de la première grammaire imprimée " à la demande de la population de Vilna », éditée à Vilna en 1586, sous le titre de Grammaire de la langue slave [Kgramatyka Sloven'ska jazyka].

Son importance réside dans l'adaptation qu'il réalise du cadre terminologique grec en slavon. Comme nous l'avons mentionné plus haut, le traité initial s'intitulait Des huit parties de l'énoncé. On notera que le terme slovo [énoncé], retenu pour traduire logos, est un terme large, utilisé dans les chroniques (cf. Le dit de la troupe d'Igor, ou Dit de la campagne d'Igor [Slovo o polku Igoreve]). Il s'agit d'un usage ancien, le terme slovo désignant dans la langue d'aujourd'hui le mot. Suivent la liste et les définitions des parties de l'énoncé :

Il y a huit parties de l'énoncé, tel que nous le parlons et l'écrivons, en dehors d'elles, il n'y a rien. Elles sont: le nom (imja), le verbe (reč'), le participe (pričastie), l'article (različie), le pronom (mesto imeni), la préposition (predlog), l'adverbe (narečie), la conjonction (souz). Et certaines de ces parties connaissent des divisions, comme le nom qui se divise en trois : le masculin, le féminin et le neutre. (Jagič 1968 [1896] : 41)

La partition grecque de l'énoncé est ici respectée, ainsi que l'ordre d'énumération des parties. Le verbe, reč', calque le rhema du grec. On note que ce terme sera amené à disparaître, cédant la place à glagol. Mais on perçoit d'emblée un problème d'adéquation aux faits de langue slave, c'est le hiatus que constitue l'article, ici traduit različie [littéralement, la distinction, et non l'articulation, qui, elle, aurait été le calque d'arthron]. Si le terme convient bien pour désigner la distinction de genre du nom, la catégorie n'est pas appropriée à la structure de la langue, qui marque le genre des noms par la désinence finale, comme en latin. Cette partie du traité se clôt d'ailleurs sur la constatation que c'est la fin du mot qui indique le genre : "Les mots masculins se terminent par un jer, les féminins par $a z$, les neutres par on.»

Ce cas est intéressant, car il met en évidence la force du modèle, incapable de trouver sa justification dans la langue décrite. Cette catégorie de l'article, inadéquate, finira logiquement par être évincée, comme nous le verrons plus loin, 
mais après avoir été maintenue artificiellement en vie par fidélité au modèle grec.

Dans le Damaskin, l'appellation des catégories et leur définition se conforment à deux modèles : celui de Denys le Thrace et celui du grammairien byzantin Choeroboscos, suivant Apollonius Dyscole. Ainsi, si la définition du verbe donnée ci-dessous reprend à Choeroboscos (cf. Grammatici graeci IV/II : 3, 22, cité dans Lallot 1998 : 162), la présentation des accidents du verbe suit l'ordre de Denys (cf. $i b i d$.) :

Le verbe est une partie de phrase non casuelle, qui nous dit la personne et le temps, l'actif et le passif ou les deux à la fois, quelle est la personne qui agit ou subit et en quel temps. Le verbe exprime cela, car il distingue les personnes et les temps, l'action et la passion ou les deux à la fois, et il montre de quelle personne est l'action. Voilà ce que dit le verbe. (Jagič,1968 [1896] : 43-44)

Dans ces traités anciens se dessine le patron de la grammaire slavonne, avec ses contours, ses formes et ses termes. Entre le Damaskin et le début du $\mathrm{XVI}^{\mathrm{e}}$ siècle, l'activité linguistique ne fournit pas de travaux d'ampleur, sinon des abécédaires. Et c'est au $\mathrm{XVI}^{\mathrm{e}}$ siècle que ce modèle se trouvera tour à tour amendé par une hybridation avec la grammaire latine qu'offrira le Donat slavon, mais aussi renforcé par la diffusion d'une grammaire hellénoslave, élaborée pour les étudiants de l'école de la confrérie laïque de Lvov, pour être enfin stabilisé dans une forme assez proche de sa forme actuelle au début du XVII ${ }^{\mathrm{e}}$ siècle.

\section{UNE ADAPTATION DE L'ARS MINOR DE DONAT}

La traduction russe de l'Ars minor nous est parvenue sous la forme de trois manuscrits distincts publiés par Vatroslav Jagić (Jagič 1968 [1896] : 524-623). Le manuscrit originel, auquel il est fait explicitement référence dans les trois versions recopiées, datait de 1522. Les copies datent de 1563. Cette traduction est l'œuvre du traducteur Dmitrij, dénommé « Dmitrij le traducteur de l'allemand (Dmitrij Tolmač nemeckogo jazyka), qui n'est autre que Dmitrij Gerasimov, ambassadeur $\mathrm{du}$ grand prince Basile III. Il s'agit d'une traduction réalisée à Novgorod pour faciliter l'enseignement du latin et en permettre une large diffusion, aux côtés du grec et du slavon. La grammaire, construite selon le modèle pédagogique du Donat sous forme de questions et réponses, met en comparaison les trois langues. Il présente la caractéristique de ne donner les exemples qu'en traduction russe :

Ce livre contient en abrégé les huit parties d'oraison ${ }^{13}$ (časti veščanija), les cas des noms, ainsi que leurs terminaisons. Il y a cinq cas, donc cinq terminaisons et cinq formes fléchies en grec, six en latin. Puis les règles de grammaire. Une fois ce petit ouvrage étudié, on entreprendra l'étude des commentaires grammaticaux, pris dans les quatre livres d' « Alexandre le professeur de grammaire » ainsi que chez de nouveaux maîtres ${ }^{14}$. (Jagič 1968 [1896] : 528)

Un résumé précède l'entrée en matière, qui expose la méthode d'enseignement que Louis Holtz qualifiait de « catéchisme grammatical» :

13 Nous traduisons à dessein «parties d'oraison », časti veščanija calquant partes orationis.

14 Plusieurs traductions du Doctrinal d'Alexandre de Villedieu ont vu le jour durant cette période, qui étaient adjointes au Donat. Cf. Kolesov 1991 : 219. 
Ce livre est appelé le Donat mineur (Donatus men'šoj), on y parle des huit parties d'oraison (časti veščanija ili reči), c'est-à-dire du nom, du pronom, du verbe, de l'adverbe et du nom participe, de la conjonction, de la préposition et de l'interjection. Les élèves débutants l'étudient après l'alphabet. (ibid.)

Dans ce préambule, il est fait explicitement référence au traité dit Damaskin. Dans sa présentation des parties du discours, Gerasimov a repris l'ordre du Donat, qui diffère de l'ordre grec, mais il a pris soin de mettre les termes issus du Damaskin en regard et de faire ainsi cohabiter les termes et les deux ordres de présentation :

Chez Damaskin aussi il y a le nom, le verbe, le participe, l'article, le pronom, la préposition, l'adverbe, la conjonction. (ibid.)

Ce qui donne, sous la forme d'un tableau :

Damaskin

Imja
Reč
Pričastie
Različie
Mesto imeni
Predlog
Narečie
Sojuz

$\begin{array}{lll}\text { Nom } & \text { Imja } & \text { Nom } \\ \text { Verbe } & \text { Proimenie } & \text { Pronom } \\ \text { Participe } & \text { Slovo ili Reč } & \text { Verbe } \\ \text { Article } & \text { Predlog slovu } & \text { Adverbe } \\ \text { Pronom } & \text { Pričastie slovu i imeni } & \text { Participe } \\ \text { Préposition } & \text { Sojuz } & \text { Conjonction } \\ \text { Adverbe } & \text { Predstavlenie } & \text { Préposition } \\ \text { Conjonction } & \text { Različie } & \text { Interjection }\end{array}$

Vladimir Kolesov insiste sur la nouveauté de la présentation de Gerasimov, marquée du sceau d'une réflexion linguistique sur les catégories elles-mêmes et sur leur caractérisation à la fois morphologique, sémantique et syntaxique, réflexion qui rejoint les idéaux réformateurs du XVI ${ }^{\mathrm{e}}$ siècle en Russie. Plusieurs remarques s'imposent au regard de ce qui va subsister dans la terminologie grammaticale. Tout d'abord, les termes nouveaux, calqués sur le latin, n'ont pas été conservés (proimenie 'pronom', predstavlenie 'préposition'), ni les périphrases explicatives, qui répondent sans doute à un souci pédagogique, comme predlog slovu 'préposé au verbe' pour désigner l'adverbe, ou bien pričastie slovu i imeni 'qui participe du verbe et du nom'. Le verbe reçoit une double dénomination, slovo ili reč', ce qui permet de ne pas s'éloigner complètement du modèle de Damaskin. L'article est une catégorie problématique, comme nous l'avons évoqué plus haut. Il entame ici sa disparition de la classification, ce qu'explique aisément la superfluité de cette catégorie en slavon. Le nombre de parties du discours doit rester inchangé, car il exprime une perfection, une clôture du système des catégories et une symétrie parfaite. Une catégorie vient donc en remplacer une autre.

Dans la tradition de la classification de Donat, la huitième partie du discours est l'interjection. Mais le plus étonnant est que le terme qui désignait l'article (različie) a été conservé pour désigner l'interjection, une partie du discours qui n'a rien à voir. Le rapport de la distinction (različie) avec l'interjection est très éloigné.

Ce hiatus est intéressant en soi. Kolesov le met en relation avec les intenses querelles théologiques du XVII ${ }^{\mathrm{e}}$ siècle. L'interjection désigne un mot jeté parmi 
les autres, alors que l'article défini désigne l'unité, l'unité de l'esprit, l'unité de Dieu (Kolesov 1991 : 221). Les frères Likhoude, moines grecs érudits, professeurs de l'Académie slavo-gréco-latine ${ }^{15}$ de Moscou en 1687, artisans de la restauration de l'hellénisme et de la suprématie grecque sur le parti latin dans l'enseignement, voyaient dans l'article, različie, le signe irréfutable de la proximité des langues russe et grecque, ainsi qu'un moyen de confondre les hérétiques, qui lui préféraient l'interjection. La catégorie de l'article doit donc sa survie artificielle à des positions idéologiques et religieuses. L'article apparaît comme un marqueur de fidélité au modèle grec.

\section{ADELPHOTĖS, GRAMMAIRE HELLÉNO-SLAVE}

C'est dans l'environnement intellectuel des confréries laïques, hostiles à la compromission avec Rome, que cette grammaire a vu le jour, à Lvov, où elle fut publiée en $1591^{16,17}$. Ces confréries veillent à la transmission des savoirs, grammaticaux notamment, dans un double souci d'indépendance vis-à-vis du catholicisme d'un côté et de l'église moscovite de l'autre. Fondées suite à la visite à Lvov du patriarche Joachim d'Antioche, elles bénéficiaient d'un privilège accordé par le patriarche universel Jérémie. Elles étaient représentées dans la plupart des grandes villes à population ruthène, particulièrement à Vilna, Lvov, Brest, Kiev. L'enseignement de cette grammaire s'adresse aux élèves de l'école de Lvov et, comme le souligne la préface, aux « disciples de la vraie foi », ceux qui refusent l'idée de l'uniatisme en passe de s'imposer. Il s'agit d'une grammaire " helléno-slave », dont la proximité avec la grammaire de Lascaris a été plusieurs fois soulignée (cf. Horbatsch 1973 : III ; Durovič 1995 : 23). Était-elle destinée à l'enseignement du grec, à celui du slavon ou bien des deux à la fois ? Il est difficile de le dire, car il s'agit d'une grammaire grecque avec traduction littérale slavonne en regard. Les deux langues y sont décrites en parallèle, ce qui avait pour effet, selon Mečkovskaja (1984), de poser le slavon comme une langue digne des Écritures.

Les termes suivent assez fidèlement ceux que l'on trouvait déjà dans le manuscrit dit Damaskin. L'Adelphotès stabilise et contribue à installer durablement la terminologie issue de la grammaire grecque, comme le remarque Olexa Horbatsch

15 L'établissement, fondé en 1682 sur le modèle de l'académie de Kiev par l'érudit Siméon de Polotsk, issu de cette académie, s'était d'abord appelé « École helléno-slave ».

16 Grammatika Dobroglagolivago Ellinoslovenskago Iazyka, soveršennago iskusstva osmi častij slova, ko nakazaniju mnogoimenitomu Rossijskomu rodu [Grammaire de la langue helléno-slave bien parlée, de l'art des huit parties de l'énoncé, pour l'instruction du peuple russe aux différents noms]. Lvov : impr. Bratskoj. 1591.

17 Le nouvel État polono-lituanien, issu de l'Union de Lublin de 1569, rassemble des populations différentes, qui sont dans leur majorité d'obédience catholique, mais la religion orthodoxe y est très représentée, tout particulièrement dans les provinces ruthènes. Si la confédération de Varsovie en 1573 reconnaissait officiellement la liberté de culte pour tous les membres de la noblesse, les populations orthodoxes faisaient l'objet de pressions visant à leur faire abandonner leur religion au profit du catholicisme. L’Union de Brest, proclamée en 1595, puis confirmée par le synode de Brest l'année suivante, consacre la naissance de l'église uniate, qui reconnaît l'autorité du pape et les dogmes catholiques, tout en conservant la liturgie byzantine slave. 
dans sa préface à l'édition de cette grammaire (Horbatsch 1973 : III). L'Adelphotès va exercer une influence importante sur le devenir de la grammaire slavonne. Le slavon y sert de métalangage, la terminologie s'en trouvera enrichie. Les exemples, donnés de façon systématique en grec puis dans leur traduction slavonne, reproduisent fidèlement les catégories reprises à la langue grecque, quitte à recourir, si besoin est, à des variantes phonétiques ou des allomorphes.

Il s'agit d'une grammaire ample et dense, de 182 feuillets, dont les auteurs anonymes annoncent d'emblée qu'elle est une compilation de différentes grammaires. Ceux-ci connaissaient bien la grammaire de celui qui sut si bien codifier l'enseignement grammatical, Donat, comme en témoigne la préface présentant la grammaire comme la clé de la compréhension des textes et le premier gradin des sept savoirs libres permettant d'accéder à la philosophie, la médecine et la théologie. L'ubomír Ďurovič a émis sur ce rapprochement une hypothèse intéressante : les auteurs pourraient avoir conçu cette grammaire en s'inspirant du Donat, mais en l'appliquant à la langue grecque (Ďurovič 1995 : 24).

La grammaire comprend quatre grandes parties, orthographe, étymologie, métrique et prosodie, et commence par une présentation de l'alphabet grec.

Le mot est défini comme « la plus petite partie de l'énoncé composé ». « La combinaison des mots produit la pensée complète qu'est la phrase », double caractérisation par la forme et le sens, qui rappelle la définition stoïcienne de la phrase, à savoir «composition en mots congruente exprimant une pensée complète » (cf. Lallot 1998 : 121-122, citation de Scholia Vaticana à la Technè, Grammatici Graeci I/III, 214, 4).

Il y a huit parties de la phrase : l'article (različie), le nom (imja), le pronom (mestoimenie), le verbe (glagol), le participe (pričastie), la préposition (predlog), l'adverbe (narečie), la conjonction (sojuz).

Cinq sont fléchies : l'article, le nom, le pronom, le verbe, le participe.

Trois ne le sont pas : la préposition, l'adverbe, la conjonction. (Horbatsch $1973: 11)$

L'article apparaît en première place. C'est une partie du discours fléchie, en genre, en nombre et en cas. C'est le pronom/adjectif démonstratif de l'objet éloigné toj qui sert de paradigme à l'article. Il est pourtant déjà en voie de marginalisation à l'époque, cantonné à la détermination de l'antécédent du relatif, comme c'est le cas en russe moderne. On peut voir une marque de fidélité au grec également dans la partie de la grammaire consacrée au verbe et à ses accidents, voix, modes et temps, qui est entièrement traduite en slavon, mais consacrée uniquement à la description du verbe grec. Si le slavon sert de métalangage, les paradigmes des conjugaisons sont ceux du grec.

La description grammaticale est poussée dans les détails, ce qui a pour effet d'élargir par calque du grec les termes disponibles pour les catégories et leurs accidents ${ }^{18}$. Comme l'indique Lubomír Ďurovič, la dette terminologique des grammaires à venir envers l'Adelphotès est immense. 
Nous passerons rapidement sur les grammaires suivantes, en dépit de leur importance dans l'histoire des grammaires. Celles-ci ont fait l'objet d'excellentes rééditions critiques dans les années 1970, ce qui a permis une foison de nouvelles études. Ces éditions sont aujourd'hui épuisées et sont remplacées petit à petit par des éditions numériques des textes, mais privées de leur important appareil critique. Parmi celles-ci, il nous faut mentionner la Grammatika slovenska (Friedhof, 1972 [1596]), grammaire de l'archiprêtre lituanien Laurent Zizanius ${ }^{19}$, professeur à l'école des confréries orthodoxes de Lvov jusqu'en 1592, puis Brest et Vilna, grammaire éditée par la confrérie de Vilna en 1596. Zizanius est aussi l'auteur d'un catéchisme jugé par les autorités orthodoxes moscovites, non conforme au dogme. La Grammatika slovenska se présente comme un manuel d'enseignement du slavon, inspiré de la lignée des grammaires grecques de Lascaris, de Melanchthon et d'Adelphotès.

Les quatre parties de la grammaire, orthographe, métrique, prosodie, étymologie, sont désignées par leur nom grec, puis dans un commentaire (tolkovanie) sont donnés les noms slaves. À la suite de la présentation des parties du discours, article (ibid.: 18), nom (ibid.: 19), pronom (ibid. : 42), verbe (ibid. : 51) y compris participe, préposition (ibid. : 78), adverbe (ibid. : 81), conjonction (ibid. : 84), sont présentés les canons de l'orthographe et du mètre (ibid.). L'ouvrage contient en outre un «Commentaire de la prière que notre Seigneur Jésus Christ enseigna à ses disciples » (Tolkovanie molitvy...).

Cette grammaire conserve l'article, en choisissant pour exemple le relatif $i z ̌ e$. Le système du verbe est repris presque intégralement d'Adelphotès. La forme du dialogue par questions et réponses, caractéristique de l'Ars minor de Donat, fait son apparition, mais de façon non systématique. Zizanius tente une synthèse de la lignée grecque et de la lignée latine en donnant de temps à autre deux termes, calquant l'un le grec et l'autre le latin, comme c'est le cas pour la description des modes verbaux. Au titre des innovations qu'apporte cette grammaire par rapport à Adelphotès, on note l'identification du vocatif (zvatel'nyj) et de l'instrumental (tvoritel'nyj), distingué de l'ablatif.

Enfin, c'est dans la grammaire de Smotrickij (1618) Grammatiki slavenskija pravilnoe syntagma osmi častij potščaniem mnogogrešnago mnixa Meletija Smotrickogo [Structure correcte des huit parties de la grammaire slave par le soin du peccamineux moine Meletius Smotrickij] que s'opéra l'alliance la plus achevée des deux lignées grammaticales grecque et latine en une véritable description des faits de langue slavons. Meletij Smotrickij est un prêtre et pédagogue ukrainien, formé à l'école d'Ostrog puis au collège jésuite de Vilna, qui a fréquenté les universités de Leipzig et Vittenberg. Il est l'auteur d'un ouvrage polémique contre l'uniatisme et l'Église catholique romaine. Prêtre orthodoxe, il a rallié néanmoins l'Église uniate lors d'une période de répression des orthodoxes dans les territoires occidentaux, à l'instar de nombreux érudits. Il a été professeur à l'école fondée par Constantin Ostrog, la plus importante école dépendant des

19 Il apparaît également sous le nom de Zizanij Lavrentij Tustanovskij Grammatika slovenska soveršennago iskustva os'mi časty slova i inyx nuždnyx [Grammaire slave de l'art correct des huit parties du mot et autres choses utiles]. 
confréries laïques d'obédience orthodoxe, qui publieront sa Grammatiki slavenskija en 1618 (cf. Horbatsch 1964 et 1974). Cette grammaire a exercé une influence considérable, a connu maintes rééditions, adaptations et refontes, et a même servi de grammaire dans tout le monde slave orthodoxe jusqu'à la diffusion des grammaires vernaculaires dans les différents pays. Dans ce dernier cas, elle devait être médiocrement adaptée aux faits de langue décrits, mais elle offrait son cadre explicatif, sa métalinguistique.

Ses très nombreux exemples sont conformes en général à ceux de la grammaire grecque, dont elle propose une traduction slavonne; la présentation des paradigmes y est très pédagogique, avec de nombreux tableaux de flexion nominale ou verbale, le regroupement des formes temporelles d'après leur morphologie, etc. Quelle que soit la partie du discours traitée, la grammaire entre dans les détails, cherche à embrasser largement le matériau de la langue slavonne. L'ordre des parties du discours se rapproche du Donat, avec le nom en première place, suivi du pronom. Le verbe ne vient qu'après, lui-même suivi du participe. L'interjection s'impose, avec une définition correspondant à son véritable usage dans la langue. Le terme choisi, calquant le terme latin (meždometie), s'installe désormais dans la grammaire.

\section{LE LEGS À LA MODERNITÉ}

$\mathrm{Au}$ XVIII ${ }^{\mathrm{e}}$ siècle, alors que s'élabore la norme de la langue russe, langue commune unifiée et plurifonctionnelle, est réexaminé le lien ancien de la langue des ancêtres avec le grec. Mixail Lomonosov, que la postérité a élevé au rang de «première université » russe à lui seul ${ }^{20}$, reconnaît volontiers la dette de la langue russe cultivée envers la langue grecque. Dans sa Préface sur l'utilité des livres d'église dans la langue russienne (Lomonosov 1952 [1758] : 587), il évoque les temps reculés où le peuple slave ne connaissait pas l'usage de l'écrit pour représenter ses pensées et où sa langue manquait des mots et expressions indispensables. Ces moyens linguistiques, c'est à la culture grecque que le peuple slave les doit : « Nous avons acquis cette richesse en même temps que la loi chrétienne grecque, lorsque les livres religieux furent traduits en slave pour la louange de Dieu. La parfaite beauté, la richesse, l'importance et la puissance du verbe grec sont très hautement estimées, ce dont témoignent volontiers les amateurs des belleslettres. » Il n'est pas seulement question ici des auteurs antiques, auxquels Lomonosov rend hommage, mais aussi, et surtout, des textes traduits du grec en slave, qui ont enrichi la langue slave. Il y a bien eu, tout de même, des traits grecs qui ont été ainsi importés dans la langue, mais avec le temps, l'oreille a fini par

20 On connaît bien la sentence de Pouchkine à propos de Lomonosov, devenue un lieu commun en Russie : «Il a fondé la première université. Pour dire mieux, il a été notre première université. » De fait, Mixail Lomonosov (1711-1765) est le prototype du savant russe, esprit encyclopédique, formé à l'Académie slavo-gréco-latine de Moscou, où il a étudié comparativement le slavon et le russe, mais aussi les langues anciennes, grec et latin. Il a fait partie des élèves qui ont été envoyés pour complément de formation en Allemagne, où il a fréquenté l'université de Marbourg. Membre de l'Académie impériale des Sciences, il a été professeur de chimie, auteur d'ouvrages d'histoire, de physique, de chimie et d'ouvrages linguistiques, dont une rhétorique et une grammaire. 
s'y habituer et les intégrer. C'est la parenté entre ces deux langues qui a permis cette assimilation. A contrario, Lomonosov insiste alors sur l'influence néfaste du latin, qui a perverti l'allemand (ibid.: 588)! On remarque au passage la distinction très nette qu'il établit entre Slaves occidentaux et Slaves orientaux, en identifiant la distinction entre une Slavia romana et une Slavia orthodoxa, opposition qui structurera maints travaux menés sur ces corpus slavons au $\mathrm{XX}^{\mathrm{e}}$ siècle (cf. Picchio 1963 ; Picchio et al. 1984).

Dans sa Grammaire russienne (Rossijskaja Grammatika), Lomonosov reprend les huit parties du discours avec leur dénomination, en modifiant l'ordre de présentation par rapport à la grammaire slavonne de Smotrickij et en réaffirmant la primauté des deux parties du discours essentielles, le nom et le verbe, revenant ainsi au modèle grec ${ }^{21}$ (Lomonosov 1952 [1755] : 408). Mais il rejette vigoureusement l'idée que l'article ${ }^{22}$ puisse accéder au statut de partie du discours :

Ces parties du discours, deux essentielles et six auxiliaires ou accessoires, doivent être impérativement dans toute langue, davantage, elles sont en surplus. Et bien que les grammaires grecques en comptent neuf, en ajoutant ő $\rho \theta \rho o v$, l'article, $\dot{o}, \dot{\eta}$, $\tau$ ó, celui-ci appartient sans aucun doute au pronom et ne peut plus constituer une partie du discours particulière, comme chez les Allemands der, die, das, ou chez les Français $l e, l a^{23}$.

Pour conclure, nous présentons une représentation comparative des termes désignant les parties du discours dans les grammaires du slavon, puis dans la grammaire de Lomonosov. L'ordre d'apparition des catégories dans les différentes grammaires y est respecté. Le recul qu'offre l'étude de la terminologie grammaticale russe sur le long terme donne l'image d'une remarquable continuité. Si elle puise à deux sources grammaticales, parentes et complémentaires, la grecque et la latine, le fonds grec a constitué, nous l'avons vu, un socle aussi ancien que durable pour un faisceau de raisons qui relèvent de l'histoire et de la culture religieuse. La proximité des langues elles-mêmes, du slavon et du grec, a pu être entretenue par les traductions de textes savants et religieux, mais la force et la plasticité du modèle descriptif et de sa terminologie, adaptable par calque, ont contribué à ce fait: la grammatisation du slavon, puis du russe, s'est faite selon le modèle de la « grammaire grecque étendue ».

21 Cf. Lallot 1997 ; Apollonius Dyscole, Syntaxe, I §13-18.

22 La survivance de l'article concerne bien sûr le latin, langue sans article. Bernard Colombat (2021 : 202), notant la persistance des catégories grecques dans la grammaire latine, choisit justement pour exemple l'article : «La langue latine ne comporte pas d'article, ce qui n'a pas empêché les Latins de reconnaître un articulus ou un pronomen articulare qui ouvrait une voie royale à l'article français. C'est en cela que l'on peut parler de grammaire étendue. L'article n'est pas une catégorie obligatoire des langues. »

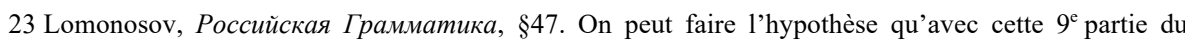
discours, Lomonosov revient ici à la tradition grecque après un détour par la tradition latine. De façon générale, il aime à montrer que le système de la langue russe est plus régulier, plus symétrique et plus concis que celui de la langue grecque. Ainsi, dans sa description du verbe, enfle-t-il le système des temps grecs, en incluant deux aoristes et trois futurs, pour mieux en dénoncer le surnombre : "Quelques langues connaissent le surnombre au contraire, puisque les verbes grecs embrassent jusqu'à neuf temps... ». Cf. Lomonosov, Российская Грамматика, §66 (1952 [1755] : 414); Archaimbault 1999 : 121-122. 
Tableau 2 - Termes désignant les parties du discours dans les grammaires du slavon, puis du russe

\begin{tabular}{l|cc|c|c|c|c}
\hline Modèle grec & $\begin{array}{c}\text { Damaskin } \\
\text { (xve siècle) }\end{array}$ & $\begin{array}{c}\text { Adelphotès } \\
(1591)\end{array}$ & $\begin{array}{c}\text { Donat } \\
\text { (Ive siècle) }\end{array}$ & $\begin{array}{c}\text { Donat slavon } \\
\text { (xve siècle) }\end{array}$ & $\begin{array}{c}\text { Smotrickij } \\
(1618)\end{array}$ & $\begin{array}{c}\text { Lomonosov } \\
\text { (1755) }\end{array}$ \\
\hline $\begin{array}{l}\text { Onoma } \\
\text { (nom) }\end{array}$ & $\begin{array}{c}\text { Imja } \\
\text { (nom) }\end{array}$ & $\begin{array}{c}\text { Različie } \\
\text { (article) }\end{array}$ & Nomen & $\begin{array}{c}\text { Imja } \\
\text { (nom) }\end{array}$ & $\begin{array}{c}\text { Imja } \\
\text { (nom) }\end{array}$ & $\begin{array}{c}\text { Imja } \\
\text { (nom) }\end{array}$ \\
\hline $\begin{array}{l}\text { Rhema } \\
\text { (verbe) }\end{array}$ & $\begin{array}{c}\text { Reč } \\
\text { (verbe) }\end{array}$ & $\begin{array}{c}\text { Imja } \\
\text { (nom) }\end{array}$ & Pronomen & $\begin{array}{c}\text { Proimenie } \\
\text { (pronom) }\end{array}$ & $\begin{array}{c}\text { Mestoimenie } \\
\text { (pronom) }\end{array}$ & $\begin{array}{c}\text { Glagol } \\
\text { (verbe) }\end{array}$ \\
\hline $\begin{array}{l}\text { Metoche } \\
\text { (participe) }\end{array}$ & $\begin{array}{c}\text { Pričastie } \\
\text { (participe) }\end{array}$ & $\begin{array}{c}\text { Mestoimenie } \\
\text { (pronom) }\end{array}$ & Verbum & $\begin{array}{c}\text { Slovo ili Reč } \\
\text { (verbe) }\end{array}$ & $\begin{array}{c}\text { Glagol } \\
\text { (verbe) }\end{array}$ & $\begin{array}{c}\text { Mestoimenie } \\
\text { (pronom) }\end{array}$ \\
\hline $\begin{array}{l}\text { Arthron } \\
\text { (article) }\end{array}$ & $\begin{array}{c}\text { Različie } \\
\text { (article) }\end{array}$ & $\begin{array}{c}\text { Glagol } \\
\text { (verbe) }\end{array}$ & Adverbium & $\begin{array}{c}\text { Predlog alovu } \\
\text { (préposé au verbe) }\end{array}$ & $\begin{array}{c}\text { Pričastie } \\
\text { (participe) }\end{array}$ & $\begin{array}{c}\text { Pričastie } \\
\text { (participe) }\end{array}$ \\
\hline $\begin{array}{l}\text { Antonymia } \\
\text { (pronom) }\end{array}$ & $\begin{array}{c}\text { Mesto imeni } \\
\text { (pronom) }\end{array}$ & $\begin{array}{c}\text { Pričastie } \\
\text { (participe) }\end{array}$ & Participium & $\begin{array}{c}\text { Pričastie slovu i imeni } \\
\text { (part. du v. et du n.) }\end{array}$ & $\begin{array}{c}\text { Predlog } \\
\text { (préposition) }\end{array}$ & $\begin{array}{c}\text { Narečie } \\
\text { (adverbe) }\end{array}$ \\
\hline $\begin{array}{l}\text { Prothesis } \\
\text { (préposition) }\end{array}$ & $\begin{array}{c}\text { Predlog } \\
\text { (préposition) }\end{array}$ & $\begin{array}{c}\text { Predlog } \\
\text { (préposition) }\end{array}$ & Coniunctio & $\begin{array}{c}\text { Souz } \\
\text { (conjonction) }\end{array}$ & $\begin{array}{c}\text { Sojuz } \\
\text { (conjonction) }\end{array}$ & $\begin{array}{c}\text { Predlog } \\
\text { (préposition) }\end{array}$ \\
\hline $\begin{array}{l}\text { Epirrhema } \\
\text { (adverbe) }\end{array}$ & $\begin{array}{c}\text { Narečie } \\
\text { (adverbe) }\end{array}$ & $\begin{array}{c}\text { Narečie } \\
\text { (adverbe) }\end{array}$ & Præpositio & $\begin{array}{l}\text { Predstavlenie } \\
\text { (préposition) }\end{array}$ & $\begin{array}{c}\text { Narečie } \\
\text { (adverbe) }\end{array}$ & $\begin{array}{c}\text { Sojuz } \\
\text { (conjonction) }\end{array}$ \\
\hline $\begin{array}{l}\text { Syndesmos } \\
\text { (conjonction) }\end{array}$ & $\begin{array}{l}\text { Sojuz } \\
\text { (conjonction) }\end{array}$ & $\begin{array}{c}\text { Sojuz } \\
\text { (conjonction) }\end{array}$ & Interiectio & $\begin{array}{c}\text { Različie/Article } \\
\text { (interjection) }\end{array}$ & $\begin{array}{l}\text { Meždometie } \\
\text { (interjection) }\end{array}$ & $\begin{array}{l}\text { Meždumetie } \\
\text { (interjection) }\end{array}$ \\
\hline
\end{tabular}

\section{BIBLIOGRAPHIE}

Archaimbault, Sylvie. 1998. Notices des grammaires du slavon et du russe. Corpus des textes linguistiques fondamentaux [http://ctlf.ens-lyon.fr, consulté le 21/03/2021].

Archaimbault, Sylvie. 1999. Préhistoire de l'aspect verbal. L'émergence de la notion dans les grammaires russes. Paris : CNRS Éditions.

Arrignon, Jean-Pierre. 2008. Chronique de Nestor. Naissance des mondes russes. Toulouse : Anacharsis.

Azam, Olivier. 2001. L'histoire controversée de la naissance du premier alphabet slave. Slavica Occitania $12: 49-89$.

Breuillard, Jean \& Stéphane Viellard. 2015. Histoire de la langue russe, des origines au XVIII' siècle. Paris : Institut d'études slaves.

Colombat, Bernard. 2021. Note sur l'interprétation à donner à la notion de « grammaire étendue ». Ad Placitum, Pour Irène Rosier-Catach, textes réunis par Laurent Cesalli, Frédéric Goubier, Anne Grondeux, Aurélien Robert, Luisa Valente : 201-206. Rome : Aracne editrice.

Comtet, Roger. 1999. La Grammatica Russica de Heinrich Wilhelm Ludolf comme grammaire piétiste. Slavica Occitania 9 : 75-98.

- 2002. Le latin des Lumières en Russie. Slavica Occitania 15 : 225-274.

Ďurovič, Lubomír. 1995. Émergence de la pensée grammaticale en Russie ancienne et formation de la grammaire du russe normatif. Histoire Épistémologie Langage $17(2): 17-32$. 
Fenne, Tönnies. 1970 [1607]. Low German Manual of Spoken Russian, Pskov, 1607, vol. 2 : Transliteration and Translation. Éd. par Louis L. Hammerich \& Roman Jakobson. Copenhague : Det Kongelige Danske Videnskabernes Selskab.

Friedhof, Gerd, éd. 1972 [1596]. L. Zizanij, Grammatika slovenska. Francfort-sur-leMain : Kubon \& Sagner.

Horbatsch, Olexa. 1964. Die Vier Ausgaben der kirckenslavischen Grammatik von M. Smotryćkyj (Frankfurter Abhandlungen zur Slavistik, 7). Wiesbaden: O. Harrassowitz.

— éd. 1973. Adelphotes, Die erste gedruckte griechisch-kirchenslavische Grammatik L'viv-Lemberg 1591. Francfort-sur-le-Main \& Munich : Kubon \& Sagner.

—éd. 1974. Smotrickij, Meletij Hrammatiki Slavenskija Pravilnoe Syntagma. Munich : Kubon \& Sagner.

Jagič, Vatroslav A. 1968 [1896]. Codex Slovenicus Rerum Grammaticarum. Pacсуждения южнославянской и русской старины о церковно-славянском языке [Réflexions de l'Antiquité slave du Sud et russe sur le slavon] (Slavische Propyläen, 25). Munich : Wilgelm Fink (publication originale : Saint-Pétersbourg. 1896).

Knjazevskaja, Olga A., éd. et trad. 1999 [XI ${ }^{\mathrm{e}}$ siècle]. Житие Мефодия [Vie de Méthode] [http://lib.pushkinskijdom.ru/Default.aspx?tabid=2164\#_ednref28, consulté le 23/03/2021].

Kolesov, Vladimir V. 1991. Развитие лингвистических идей у восточных славян эпохи Средневековья [Épanouissement des idées linguistiques chez les slaves orientaux au Moyen-Âge]. История лингвистических учений [Histoire des savoirs linguistiques], vol. 4 : Позднее Средневековье [Fin du Moyen Âge], dir. par Agnja V. Desnickaja, 208-254. Saint-Pétersbourg : Наука.

Kuz'minova, Elena A. 2002. Annali del Istituto Universitario Orientale di Napoli, Slavistica 1 (Грамматический сборник 1620-х г2. [Recueil grammatical des années 1620]).

Lallot, Jean. 1997. Apollonius Dyscole. De la construction (syntaxe). 2 vol. Paris : Vrin.

- 1998. La grammaire de Denys le Thrace. $2^{\mathrm{e}}$ éd. Paris : CNRS Éditions.

Lomonosov, Mixail V. 1952 [1758]. Предисловие о пользе книг церковных в Российском языке [Préface sur l'utilité des livres d'église dans la langue russienne]. Полное собрание сочинений. Труды по филологии 1739-1758 г2 [Euvres complètes. Travaux philologiques], vol. 7, 585-592. Moscou \& Leningrad : Издательство Академии Наук СССР.

- 1952 [1755]. Российская Грамматика [Grammaire russienne]. Полное собрание сочинений. Труды по филологии 1739-1758 г2 [CEuvres complètes, Travaux philologiques], vol. 7, 388-578. Moscou \& Leningrad : Издательство Академии Наук СССР.

Ludolf, Heinrich Wilhelm. 1959 [1696]. Henrici Wilhelmi Ludolfi Grammatica russica. Éd. par Boris Ottokar Unbegaun. Oxford : Clarendon Press.

Mečkovskaja, Nina B. 1984. Ранние восточнославянские грамматики [Premières grammaires slaves de l'Est]. Dir. par Adam E. Suprun. Minsk : Изд. Университетское. 
Meillet, Antoine. 1910. L'unité des langues slaves. La Revue de Paris, $1^{\text {er }}$ févr. 1910.

Picchio, Riccardo. 1963. A proposito della Slavia ortodossa e della comunità linguistica slava ecclesiastica. Ricerche slavistiche 11 : 105-127.

—, Harvey Goldblatt \& Susanne Fusso, éd. 1984. Aspects of the Slavic Language Question. 2 vol. Columbus, Ohio : Slavica.

Ridley, Mark. 1996 [1594-1599]. A dictionarie of the vulgar Russe tongue. Attribué à Mark Ridley, éd. à partir de manuscrits de la fin du XVI ${ }^{\mathrm{e}}$ siècle et avec une introduction par Gerald Stone. Cologne : Böhlau Verlag.

Sohier, Jean. 1987 [1724]. Grammaire \& methode russes et françoises. Éd. en facsimilé par Boris A. Uspenskij. 2 vol. Munich : Verlag Otto Sagner.

Uspenskij, Boris A. 1994. Краткий очерк истории русского литературного языка (XI-XIX вв.) [Abrégé d'histoire de la langue russe normée $\left(\mathrm{XI}^{\mathrm{e}}\right.$ XIX ${ }^{\mathrm{e}}$ siècles)]. Moscou : Гнозис.

- 1997. Избранные труды [Euvres complètes], vol. III : Общее и славянское языкознание [Linguistique générale et linguistique slave]. $2^{\mathrm{e}}$ éd. Moscou : Языки русской культуры.

- 2002. История русского литературного языка (XI-XVII вв.) [Histoire de la langue russe normée ( $\mathrm{XI}^{\mathrm{e}}-\mathrm{XVII}{ }^{\mathrm{e}}$ siècles)]. $3^{\mathrm{e}}$ éd. Moscou : Аспект Пресc.

Vaillant, André. 1968. Textes vieux slaves. 2 vol. Paris : Institut d'études slaves.

Worth, Dean. 1983. The Origins of Russian Grammar. Notes on the State of Russian Philology before the Advent of Printed Grammars (UCLA Slavic Studies, 5). Columbus, Ohio : Slavica.

Živov, Viktor M. 2017. История языка русской письменности [Histoire de la langue russe écrite]. 2 vol. Moscou : Университет Дмитрия Пожарского. 